TRANSACTIONS OF THE

AMERICAN MATHEMATICAL SOCIETY

Volume 363, Number 6, June 2011, Pages 2849-2863

S 0002-9947(2011)05015-4

Article electronically published on January 25, 2011

\title{
ON THE WEAK KÄHLER-RICCI FLOW
}

\author{
X. X. CHEN, G. TIAN, AND Z. ZHANG
}

\begin{abstract}
In this paper, we define and study the Kähler-Ricci flow with initial data not being smooth and discuss some natural applications.
\end{abstract}

\section{INTRODUCTION}

Let $M$ be an $n$-dimensional compact (without boundary) Kähler manifold. A Kähler metric $g$ can be represented by its Kähler form $\omega_{g}$ on $M$. Consider the Kähler-Ricci flow on $M \times[0, T)$ :

$$
\frac{\partial \omega_{g(t)}}{\partial t}=-\operatorname{Ric}\left(\omega_{g(t)}\right)
$$

where $g(t)$ is a family of Kähler metrics and Ric $\left(\omega_{g}\right)$ denotes the Ricci curvature of $g$. It is known that for any smooth Kähler metric $g_{0}$, there is a unique solution $g(t)$ of (1.1) for some maximal time $T>0$ with $g(0)=g_{0}$. In general, $T$ will depend on the initial metric $g_{0}$. However, in the Kähler manifold case, this only depends on the Kähler class and the first Chern class. This observation plays an important role in our introduction of weak Ricci flow over Kähler manifolds.

The Ricci flow was introduced by R. Hamilton in 20. Extensive research has been done in the case of the smooth flow (cf. [22, 6], 14], 21], 25, [12, [13, [30, or [15] for complete updated references). In order to prove the uniqueness of extremal Kähler metrics in full generality, in [9, the first two named authors were led to the study of Kähler-Ricci flows in the weak sense. More precisely, we proved in [9] that for any initial $L^{\infty}$-bounded Kähler metric, there is a uniformly $L^{\infty}$-bounded solution of (1.1) with the given initial "metric" in a suitable sense. Moreover, the volume form of the solution converges strongly to the volume form of the initial Kähler "metric" in $L^{2}$-topology as $t \rightarrow 0$.

As one may expect, such a weak solution of (1.1) should become smooth immediately after $t>0$. Indeed, we confirm this in this paper in a more general setting.

Theorem 1.1. For any Kähler current $g_{0}$ with $C^{1,1}$ bounded potential on $M$, there is a unique smooth solution $g(t)(t \in(0, T))$ of (1.1) such that $\lim _{t \rightarrow 0^{+}} g(t)=g_{0}$ in a $C^{1, \alpha}(\forall \alpha \in(0,1))$ norm at the potential level.

Received by the editors October 9, 2008.

2000 Mathematics Subject Classification. Primary 53C25; Secondary 53C99, 58J99.

Key words and phrases. Differential geometry, geometric evolution equation.

The first author was supported in part by NSF funds.

The second author was supported in part by NSF funds.

(C)2011 American Mathematical Society Reverts to public domain 28 years from publication 
A special case of Riemann surfaces was also considered in 11 with a completely different proof.

It was known that any Kähler metric with constant scalar curvature is the absolute minimizer of the K-energy on the space of all Kähler metrics with a fixed Kähler class ([8, 18, 24 and [9] 1$]$. From an analytic point of view, it is an extremely difficult problem to prove the existence of a Kähler metric with constant scalar curvature in general cases. One approach is to construct weak minimizers of the K-energy by applying certain variational or continuous methods. This seems to be a plausible direct approach but very hard. However, even if we obtained a $C^{1,1}$ minimizer of the K-energy functional, we would still face the regularity problem. In [8], the first named author made the following conjecture: Any $C^{1,1}$ minimizers of the K-energy in a given Kähler class must be smooth. As a consequence of the above theorem, we can solve this conjecture in canonically polarized cases.

Corollary 1.2. In a Kähler class which is proportional to the first Chern class, any $L^{\infty}$ Kähler metric which minimizes the K-energy functional must be smooth.

There is another motivation for this short paper. On a general Kähler manifold, the Kähler-Ricci flow (1.1) may develop singularity in finite time (see 31]). In an on-going project with his collaborators, the second named author proposed some problems of studying how the Kähler-Ricci flow extends across the finite time singularity (see 28, for more discussion). One of the problems involves constructing solutions of the Kähler-Ricci flow with much weaker initial metrics, possibly on spaces with mild singularity. Our second result gives a partial solution to this problem.

First we recall some standard facts: by the Hodge Theorem, the space of all Kähler metrics in a fixed Kähler class given by a smooth Kähler metric $\omega$ is

$$
P(M, \omega)=\left\{\varphi \in C^{\infty}(M) \mid \omega_{\varphi}=\omega+\sqrt{-1} \partial \bar{\partial} \varphi>0 \text { on } M\right\} .
$$

We denote by $C l_{L^{\infty}} P(M, \omega)$ all bounded functions plurisubharmonic with respect to $\omega$. Then for any $\varphi \in C l_{L^{\infty}} P(M, \omega)$, there is a well-defined (Borel) measure $(\omega+\sqrt{-1} \partial \bar{\partial} \varphi)^{n} \geq 0$ using the understanding from pluripotential theory about Monge-Ampère operators introduced in 2. One can also think of this as a volume form in a weak sense. Now we can state our second result.

Theorem 1.3. For any Kähler potential $\varphi_{0} \in C l_{L^{\infty}} P(M, \omega)$ whose volume form is $L^{p}(M, \omega)(p \geq 3)$, there is a unique smooth solution $g(t)$ of (1.1) for $t \in(0, T)$ such that $\frac{\omega_{g(t)}^{n}}{\omega^{n}}$ converges to $\frac{\omega_{\varphi_{0}}^{n}}{\omega^{n}}$ strongly in the $L^{2}$-topology.

The organization of this paper is as follows: in Section 2, we show the existence of the weak solution for the induced potential flow which is equivalent to the KählerRicci flow. $C^{0}$-estimates will be derived for the solution and its time derivative. We also examine a convergence problem for these weak solutions as $t$ tends to 0 . In Section 3, we derive the 2 nd and 3 rd order estimates for the weak solutions. Then Theorems 1.1 and 1.3 follow. In the last section, we prove Corollary 1.2.

\section{WeAK SOlutions of the KÄHLER-RICCI FLOW}

First we reduce the Kähler-Ricci flow (1.1) to a scalar flow. To start with, we note that $\left[\omega_{g_{0}}\right]-t c_{1}(M)$ represents a Kähler class whenever $0 \leq t \leq T$ for a

\footnotetext{
${ }^{1}$ For Kähler-Einstein metrics, cf. [1], 17] and 29].
} 
sufficiently small $T>0$ depending only on $\left[\omega_{g_{0}}\right]$ and $c_{1}(M)$. Choose a smooth family of Kähler forms $\omega_{t}$ for $t \in[0, T]$ such that $\omega_{0}=\omega$ and $\left[\omega_{t}\right]=\left[\omega_{g_{0}}\right]-t c_{1}(M)$. Write $\omega_{g(t)}=\omega_{t}+\sqrt{-1} \partial \bar{\partial} \varphi(t)$. Then, one can show by standard argument that $g(t)$ solves (1.1) if and only if $\varphi(t)$ solves the following scalar flow:

$$
\frac{\partial \varphi}{\partial t}=\log \frac{\left(\omega_{t}+\sqrt{-1} \partial \bar{\partial} \varphi\right)^{n}}{\omega_{t}^{n}}-h_{\omega_{t}},
$$

where $h_{\omega_{t}}$ is defined by

$$
\operatorname{Ric}\left(\omega_{t}\right)+\frac{\partial \omega_{t}}{\partial t}=\sqrt{-1} \partial \bar{\partial} h_{\omega_{t}}, \text { and } \int_{X}\left(e^{h_{\omega_{t}}}-1\right) \omega_{t}^{n}=0 .
$$

Clearly such an $h\left(\omega_{t}\right)$ does exist and is unique. As usual, we will regard either (1.1) or (2.1) as the Kähler-Ricci flow on $M$. For simplicity, we denote by $\omega_{\varphi}$ the Kähler form $\omega_{t}+\sqrt{-1} \partial \bar{\partial} \varphi$.

To construct a weak solution to the Kähler-Ricci flow equation with non-smooth initial Kähler potentials, as usual, we use smooth approximations of the initial data. Let $\varphi_{0}$ be a bounded Kähler potential such that its volume form is in $L^{p}$ for some $p>1$. This actually implies Hölder continuity of $\varphi_{0}$ from the result in [27]. Let $\varphi_{0}(s)(0 \leq s \leq 1)$ be a 1-parameter Kähler potential such that the following properties hold:

(1) $\varphi_{0}(0)=\varphi_{0}$ and $\varphi_{0}(s) \in P(M, \omega)$ for $0<s \leq 1$.

(2) If $\varphi_{0}$ has $C^{1,1}$ bound, then $\varphi_{0}(s)$ has uniform $C^{1,1}$ upper bound and $\varphi_{0}(s)(s>0) \rightarrow \varphi_{0}$ strongly in $W^{2, q}(M, \omega)$ for $q$ sufficiently large.

(3) $\varphi_{0}(s)$ converges to $\varphi_{0}$ uniformly in $L^{\infty}$-topology and the volume form ratio converges to $\frac{\omega_{\varphi_{0}}^{n}}{\omega^{n}}$ strongly in $L^{p}(p>1)$.

Later in Section 3, less regularity will be assumed, and so the approximation would also be less restrictive.

It is known (cf. 31]) that for any $\varphi$ in $P(M, \omega)$ there is a unique smooth solution of (2.1) on $[0, T]$ with $\varphi$ as the initial value. Therefore, we have $\varphi(s, t) \in P(M, \omega)$ $(0<s \leq 1, t \in[0, T])$ satisfying:

$$
\begin{aligned}
\frac{\partial \varphi(s, t)}{\partial t} & =\log \frac{\left(\omega_{t}+\sqrt{-1} \partial \bar{\partial} \varphi\right)^{n}}{\omega_{t}^{n}}-h_{\omega_{t}}, \\
\varphi(s, 0) & =\varphi_{0}(s) .
\end{aligned}
$$

Clearly, for each $s>0$ fixed, there exists a uniform $C^{2, \alpha}$ bound for $\varphi(s, t)$ $(0 \leq t \leq T)$. However, the upper bound may well depend on $s$ and so may blow up when $t, s$ are both small. If there is a limit

$$
\varphi(t)=\lim _{s \rightarrow 0} \varphi(s, t), \forall t \in[0, T],
$$

then we can regard $\varphi(t)$ as a weak solution of (2.1) with initial value $\varphi_{0}$.

Lemma 2.1. The solutions $\varphi(s, t)$ converge uniformly to a family of functions $\varphi(t)$ $(t \in[0, T])$ as $s$ tends to 0 .

Proof. For any positive $s$ and $s^{\prime}$, put $\psi(t)=\varphi\left(s^{\prime}, t\right)-\varphi(s, t)$; then we have

$$
\frac{\partial \psi}{\partial t}=\log \frac{\left(\omega_{\varphi(s, t)}+\sqrt{-1} \partial \bar{\partial} \psi\right)^{n}}{\left(\omega_{t}+\sqrt{-1} \partial \bar{\partial} \varphi(s, t)\right)^{n}}
$$


Then the Maximum Principle implies that $\sup _{M}|\psi(t)| \leq \sup _{M}|\psi(0)|$; that is,

$$
\sup _{M}\left|\varphi\left(s^{\prime}, t\right)-\varphi(s, t)\right| \leq \sup _{M}\left|\varphi_{0}\left(s^{\prime}\right)-\varphi_{0}(s)\right| .
$$

The lemma then follows easily from our choice of the initial data $\varphi_{0}(s)$.

We shall show that $\varphi(t)$ solves the Kähler-Ricci flow and consequently is a weak solution in a suitable sense. For this purpose, one needs to justify that $\varphi(t)$ is a smooth family for $t>0$ which solves (2.1) for $t>0$ such that $\lim _{t \rightarrow 0+} \varphi(t)=\varphi_{0}$. Of course, this is the core part of this paper. First we derive a few estimates on $\varphi(s, t)$ uniform for $s \in(0,1]$ (Sometimes we abbreviate $\varphi(s, t)$ as $\varphi$ for simplicity). These estimates would eventually be passed on to $\varphi(t)$ by taking the limit as $s$ tends to 0 in some proper sense.

Lemma 2.2. If $\varphi_{0}$ is bounded, then there exists a uniform constant $C$ such that for any $s \in(0,1]$ and $t \in[0, T]$,

$$
|\varphi(s, t)| \leq C .
$$

Proof. Choose $c$ such that $\left|h_{\omega_{t}}\right|_{C^{0}} \leq c$ for all $t \in[0, T]$. Applying the Maximum Principle to $\varphi(s, t) \pm c t$, we can obtain

$$
\min \varphi_{0}(s)-c t \leq \varphi(s, t) \leq \max \varphi_{0}(s)+c t, \quad \forall s>0 .
$$

Then the bound on $\varphi$ follows.

The next lemma gives an estimate on the volume form for the Kähler-Ricci flow when $t>0$. In this paper, $\Delta_{\varphi}$ always stands for Laplacian with respect to the flow metric $\omega_{\varphi}=\omega_{t}+\sqrt{-1} \partial \bar{\partial} \varphi$. Traditionally, $C$ might stand for different positive constants at different places.

Lemma 2.3. For $t>0$, the volume form has uniform upper and positive lower bounds which may depend on $t$.

Proof. Differentiating the Kähler-Ricci flow equation

$$
\frac{\partial \varphi}{\partial t}=\log \frac{\left(\omega_{t}+\sqrt{-1} \partial \bar{\partial} \varphi\right)^{n}}{\omega_{t}^{n}}-h_{\omega_{t}}
$$

we get

$$
\begin{aligned}
\varphi^{\prime \prime} & =\Delta_{\varphi} \varphi^{\prime}-h_{\omega_{t}}^{\prime}-\operatorname{tr}_{\omega_{t}}\left(\frac{\partial}{\partial t} \omega_{t}\right)+\operatorname{tr}_{\varphi}\left(\frac{\partial}{\partial t} \omega_{t}\right) \\
& \leq \Delta_{\varphi} \varphi^{\prime}+C\left(1+\operatorname{tr}_{\varphi} \omega\right) .
\end{aligned}
$$

Similarly, we have

$$
\varphi^{\prime \prime} \geq \Delta_{\varphi} \varphi^{\prime}-C\left(1+\operatorname{tr}_{\varphi} \omega\right)
$$

which will be used for the lower bound later in this proof.

For the upper bound, let us consider

$$
F_{+}=-\varphi+t \varphi^{\prime}
$$

The $t$-derivative gives

$$
\begin{aligned}
\frac{\partial}{\partial t} F_{+} & =-\varphi^{\prime}+\varphi^{\prime}+t \varphi^{\prime \prime} \\
& \leq t \Delta_{\varphi} \varphi^{\prime}+C t\left(1+\operatorname{tr}_{\varphi} \omega\right) \\
& =\Delta_{\varphi}\left(-\varphi+t \varphi^{\prime}\right)+\Delta_{\varphi} \varphi+C t\left(1+\operatorname{tr}_{\varphi} \omega\right) \\
& =\Delta_{\varphi} F_{+}+n-\operatorname{tr}_{\varphi} \omega_{t}+C t\left(1+\operatorname{tr}_{\varphi} \omega\right) \\
& \leq \Delta_{\varphi} F_{+}+C-(C-C t)\left(1+\operatorname{tr}_{\varphi} \omega\right) .
\end{aligned}
$$


For $t$ small enough, we have

$$
\frac{\partial}{\partial t} F_{+} \leq \Delta_{\varphi} F_{+}+C
$$

Since $F_{+}(0)$ is uniformly bounded from above, it follows from the Maximum Principle that $F_{+}$has a uniform upper bound.

For the lower bound, set

$$
F_{-}=\varphi+t \varphi^{\prime}=\varphi-t h_{\omega}+t \log \frac{\omega_{\varphi}^{n}}{\omega_{t}^{n}} .
$$

Then, using the same $C$ to denote different constants

$$
\begin{aligned}
\frac{\partial}{\partial t} F_{-} & =\varphi^{\prime}+\varphi^{\prime}+t \varphi^{\prime \prime} \\
& \geq 2 \varphi^{\prime}+t\left(\Delta_{\varphi} \varphi^{\prime}-C\left(1+\operatorname{tr}_{\varphi} \omega\right)\right) \\
& =\Delta_{\varphi}\left(\varphi+t \varphi^{\prime}\right)-\Delta_{\varphi} \varphi+2 \varphi^{\prime}-C t\left(1+\operatorname{tr}_{\varphi} \omega\right) \\
& =\Delta_{\varphi} F_{-}-n+\operatorname{tr}_{\varphi} \omega_{t}+2 \log \frac{\omega_{\varphi}^{n}}{\omega_{t}^{n}}-2 h_{\omega_{t}}-C t\left(1+\operatorname{tr}_{\varphi} \omega\right) \\
& \geq \Delta_{\varphi} F_{-}-C+(C-C t)\left(1+\operatorname{tr}_{\varphi} \omega\right)+2 \log \frac{\omega_{\varphi}^{n}}{\omega_{t}^{n}} \\
& \geq \Delta_{\varphi} F_{-}-C+(C-C t)\left(\frac{\omega_{\varphi}^{n}}{\omega_{t}^{n}}\right)^{-\frac{1}{n}}+2 \log \frac{\omega_{\varphi}^{n}}{\omega_{t}^{n}}
\end{aligned}
$$

It is easy to see that for $x>0$, with the understanding that these two $C$ 's are not the same,

$$
x^{-\frac{1}{n}}+C \log x \geq-C .
$$

It follows that for $t$ small, we have

$$
\frac{\partial}{\partial t} F_{-} \geq \Delta_{\varphi} F_{-}-C
$$

Then the Maximum Principle implies that $F_{-}>-C$ for some uniform constant $C$; that is, the volume form $\frac{\omega_{\varphi}^{n}}{\omega_{t}^{n}}$ has a uniform positive lower bound when considered uniformly away from initial time.

Next we exam whether $\lim _{t \rightarrow 0+} \varphi(t)=\varphi_{0}$. To make sure of this, we derive some integral estimates.

Lemma 2.4. If $\varphi_{0}$ is bounded and $\frac{\omega_{\varphi_{0}}^{n}}{\omega^{n}}$ is in $L^{p}(p \geq 1)$, then for any $s \in(0,1]$, there exists a positive function $C(t)$ of $t$ such that

$$
\left\|\frac{\omega_{\varphi}^{n}}{\omega^{n}}\right\|_{L^{p}} \leq C(t)
$$

Moreover, $C(t)$ is independent of $s$ and is uniformly bounded for $t \in[0, T]$ with $T<\infty$.

Proof. For $\lambda>0$, set the modified volume ratio as

$$
f_{\varphi}=\frac{\omega_{\varphi}^{n}}{\omega_{t}{ }^{n}} e^{-\lambda \varphi}
$$

We are considering classic smooth solutions. Let $C$ be a constant satisfying:

$$
\left|\frac{\partial}{\partial t} \omega_{t}\right|_{\omega_{t}}+\left|\operatorname{Ric}\left(\omega_{t}\right)\right|_{\omega_{t}}+|\operatorname{Ric}(\omega)|_{\omega_{t}}+\left|\partial \bar{\partial} h_{\omega_{t}}\right|_{\omega_{t}} \leq C
$$


Use $\nabla$ to denote the gradient with respect to $\omega_{\varphi}$. Then for $\lambda$ sufficiently large,

$$
\begin{aligned}
\frac{\partial \log f_{\varphi}}{\partial t}= & \operatorname{tr}_{\varphi}\left(\frac{\partial}{\partial t} \omega_{t}\right)+\triangle_{\varphi} \varphi^{\prime}-\operatorname{tr}_{\omega_{t}}\left(\frac{\partial}{\partial t} \omega_{t}\right)-\lambda \varphi^{\prime} \\
= & \Delta_{\varphi} \log f_{\varphi}+\operatorname{tr}_{\varphi}\left(\frac{\partial}{\partial t} \omega_{t}+\lambda \sqrt{-1} \partial \bar{\partial} \varphi-\sqrt{-1} \partial \bar{\partial} h_{\omega_{t}}\right) \\
& +R_{\omega_{t}}-\operatorname{tr}_{\omega_{t}} h_{\omega_{t}}-\lambda \varphi^{\prime} \\
\leq & \Delta_{\varphi} \log f_{\varphi}+\lambda-(\lambda-2 C) \cdot \operatorname{tr}_{\varphi} \omega_{t}-\lambda \log f_{\varphi}+\lambda h_{\omega_{t}}+2 C \\
\leq & \frac{\Delta_{\varphi} f_{\varphi}}{f_{\varphi}}-\frac{\left|\nabla f_{\varphi}\right|_{\varphi}^{2}}{f_{\varphi}^{2}}+2 C-\lambda \log f_{\varphi} .
\end{aligned}
$$

Thus, we have

$$
\frac{\partial f_{\varphi}}{\partial t} \leq \triangle_{\varphi} f_{\varphi}-\frac{\left|\nabla f_{\varphi}\right|_{\varphi}^{2}}{f_{\varphi}}+2 C f_{\varphi}-\lambda f_{\varphi} \log f_{\varphi}
$$

For any $p \geq 1$, we have

$$
\begin{aligned}
& \frac{d}{d t} \int_{M} f_{\varphi}^{p} e^{\lambda \varphi} \omega_{t}^{n} \\
& =p \int_{M} f_{\varphi}^{p-1} \frac{\partial f_{\varphi}}{\partial t} e^{\lambda \varphi} \omega_{t}^{n}+\int_{M} f_{\varphi}^{p} e^{\lambda \varphi} \frac{\partial \omega_{t}^{n}}{\partial t}+\lambda \int_{M} f_{\varphi}^{p} e^{\lambda \varphi} \frac{\partial \varphi}{\partial t} \omega_{t}^{n} \\
& =p \int_{M} f_{\varphi}^{p-1} \frac{\partial f_{\varphi}}{\partial t} e^{\lambda \varphi} \omega_{t}^{n}+\int_{M} f_{\varphi}^{p}\left(-\mathrm{R}_{\omega_{t}}+\operatorname{tr}_{\omega_{t}} h_{\omega_{t}}\right) e^{\lambda \varphi} \omega_{t}^{n} \\
& +\lambda \int_{M} f_{\varphi}^{p} e^{\lambda \varphi}\left(\log \frac{\omega_{\varphi}^{n}}{\omega_{t}^{n}}-h_{\omega_{t}}\right) \omega_{t}^{n} \\
& \leq p \int_{M} f_{\varphi}^{p-1}\left(\Delta_{\varphi} f_{\varphi}-\frac{\left|\nabla f_{\varphi}\right|_{\varphi}^{2}}{f_{\varphi}}+f_{\varphi}\left(2 C-\lambda \log f_{\varphi}\right)\right) e^{\lambda \varphi} \omega_{t}^{n}+C \int_{M} f_{\varphi}^{p} e^{\lambda \varphi} \omega_{t}^{n} \\
& +\lambda \int_{M} f_{\varphi}^{p} e^{\lambda \varphi}\left(\log \frac{\omega_{\varphi}^{n}}{\omega_{t}^{n}}-h_{\omega_{t}}\right) \omega_{t}^{n} \\
& \leq \int_{M} f_{\varphi}^{p-2}\left(p\left(\Delta_{\varphi} f_{\varphi}-\frac{\left|\nabla f_{\varphi}\right|_{\varphi}^{2}}{f_{\varphi}}\right)+f_{\varphi}\left(2 p C-\lambda(p-1) \log f_{\varphi}\right)\right) \omega_{\varphi}^{n} \\
& +C_{\lambda} \int_{M} f_{\varphi}^{p} e^{\lambda \varphi} \omega_{t}^{n} \\
& \leq-p(p-1) \int_{M}^{M} f_{\varphi}^{p-2}\left|\nabla f_{\varphi}\right|_{\varphi}^{2} e^{\lambda \varphi} \omega_{t}^{n}+C_{\lambda} \int_{M}\left(f_{\varphi}^{p}+f_{\varphi}^{p-1}\right) e^{\lambda \varphi} \omega_{t}^{n} .
\end{aligned}
$$

Here we have used the fact that the function $x \log x$ has a lower bound for $x>0$.

Since $\varphi$ is uniformly bounded, it follows that $\frac{\omega_{\varphi}^{n}}{\omega^{n}}$ is uniformly bounded in $L^{p}$ for all $t \in[0, T]$.

It follows from this lemma that the $L^{p}$-norm of $\varphi(t)$ is uniformly bounded. By the work of Kolodziej [27, we know that $\|\varphi(t)\|_{C^{\alpha}}$ is uniformly bounded, where $\alpha=\alpha(p)>0$ may depend on $p>1$. Then for any sequence $t_{i} \rightarrow 0$, there is a subsequence, still denoted by $t_{i}$ for simplicity, such that $\varphi\left(t_{i}\right)$ converges to a $C^{\alpha}$ function $\tilde{\varphi}_{0}$ in the $C^{\beta}$-topology for some $\beta \in(0, \alpha)$. A priori, this limit potential might well depend on the sequence we choose.

Lemma 2.5. If $p \geq 3$, we have $\tilde{\varphi}_{0}=\varphi_{0}$. Moreover, $\frac{\omega_{\varphi(t)}^{n}}{\omega^{n}}$ converges to $\frac{\omega_{\varphi_{0}}^{n}}{\omega^{n}}$ strongly in the $L^{2}$-topology, where a smooth volume form is used to define the $L^{2}$-topology and the choice clearly does not matter.

Proof. First we assume that $\varphi_{0}$ is smooth, i.e., one of $\varphi_{0}(s)(s>0)$. In the proof of the preceding lemma, set $p=3$ and we have

$$
\frac{d}{d t} \int_{M} f_{\varphi}^{3} e^{\lambda \varphi} \omega_{t}^{n} \leq-6 \int_{M} f_{\varphi}\left|\nabla f_{\varphi}\right|_{\varphi}^{2} e^{\lambda \varphi} \omega_{t}^{n}+C \int_{M} f_{\varphi}^{3} e^{\lambda \varphi} \omega_{t}^{n}+C
$$


which implies

$$
\int_{0}^{t} \int_{M} f_{\varphi}\left|\nabla f_{\varphi}\right|_{\varphi}^{2} \omega_{t}^{n} d u \leq C
$$

Now set

$$
f=\frac{\omega_{\varphi}^{n}}{\omega_{t}^{n}}
$$

Claim 1. We have for $t$ in some finite interval,

$$
\begin{aligned}
& \int_{0}^{t} \int_{M}|\nabla f|_{\varphi}^{2} \omega_{\varphi}^{n} d u=\int_{0}^{t} \int_{M} f|\nabla f|_{\varphi}^{2} \omega_{t}^{n} d u \leq C, \\
& \int_{0}^{t} \int_{M} f_{\varphi}^{2}|\nabla \varphi|_{\varphi}^{2} \omega_{\varphi}^{n} d u \leq C .
\end{aligned}
$$

Proof of Claim 1. Since $\varphi$ is uniformly bounded, we can choose a uniform constant $c$ such that $\varphi-c \leq 0$. Then we have

$$
\begin{aligned}
\int_{M} & f_{\varphi}^{2}|\nabla \varphi|_{\varphi}^{2} \omega_{\varphi}^{n} \\
= & -2 \int_{M} f_{\varphi}\left(\nabla f_{\varphi} \cdot \nabla \varphi\right)_{\varphi}(\varphi-c) \omega_{\varphi}^{n}-\int_{M} f_{\varphi}^{2}(\varphi-c) \Delta_{\varphi} \varphi \omega_{\varphi}^{n} \\
\leq & \frac{1}{2} \int_{M} f_{\varphi}^{2}|\nabla \varphi|_{\varphi}^{2} \omega_{\varphi}^{n}+C \int_{M}\left|\nabla f_{\varphi}\right|_{\varphi}^{2} \omega_{\varphi}^{n}-n \int_{M} f_{\varphi}^{2}(\varphi-c) \omega_{\varphi}^{n} \\
& +\int_{M} f_{\varphi}^{2}(\varphi-c) \operatorname{tr}_{\varphi} \omega_{t} \omega_{\varphi}^{n} \\
\leq & \frac{1}{2} \int_{M} f_{\varphi}^{2}|\nabla \varphi|_{\varphi}^{2} \omega_{\varphi}^{n}+C \int_{M}\left|\nabla f_{\varphi}\right|_{\varphi}^{2} \omega_{\varphi}^{n}+C \int_{M} f_{\varphi}^{2} \omega_{\varphi}^{n} \\
= & \frac{1}{2} \int_{M} f_{\varphi}^{2}|\nabla \varphi|_{\varphi}^{2} \omega_{\varphi}^{n}+C \int_{M} f_{\varphi}\left|\nabla f_{\varphi}\right|_{\varphi}^{2} \omega_{t}^{n}+C \int_{M} f_{\varphi}^{3} e^{\lambda \varphi} \omega_{t}^{n} .
\end{aligned}
$$

It follows by integrating over $t$ and (2.4) that

$$
\int_{0}^{t} \int_{M} f_{\varphi}^{2}|\nabla \varphi|_{\varphi}^{2} \omega_{\varphi}^{n} d u \leq C
$$

To prove the first inequality, we observe

$$
f=e^{\lambda \varphi} f_{\varphi} .
$$

Then,

$$
\begin{aligned}
\int_{M} f|\nabla f|_{\varphi}^{2} \omega_{t}^{n} & =\int_{M}\left|\nabla\left(f_{\varphi} e^{\lambda \varphi}\right)\right|_{\varphi}^{2} \omega_{\varphi}^{n} \\
& \leq 2 \int_{M}\left|\nabla f_{\varphi}\right|^{2} e^{2 \lambda \varphi} \omega_{\varphi}^{n}+2 \lambda^{2} \int_{M} f_{\varphi}^{2}|\nabla \varphi|_{\varphi}^{2} e^{2 \lambda \varphi} \omega_{\varphi}^{n} \\
& \leq C\left(\int_{M}\left|\nabla f_{\varphi}\right|_{\varphi}^{2} \omega_{\varphi}^{n}+\int_{M} f_{\varphi}^{2}|\nabla \varphi|_{\varphi}^{2} \omega_{\varphi}^{n}\right) .
\end{aligned}
$$

Thus the first inequality follows from the second one and (2.4). Claim 1 is proved.

Claim 2. For any smooth non-negative cut-off function $\chi$ (fixed), we have,

$$
\int_{M} f|\nabla \chi|_{\varphi}^{2} \omega_{t}^{n}=\int_{M}|\nabla \chi|_{\varphi}^{2} \omega_{\varphi}^{n} \leq C\left(|\nabla \chi|_{\omega, L^{\infty}}\right) .
$$

Proof of Claim 2.

$$
\begin{aligned}
\int_{M} f|\nabla \chi|_{\varphi}^{2} \omega_{t}^{n} & =\int_{M}|\nabla \chi|_{\varphi}^{2} \omega_{\varphi}^{n} \\
& \leq \int_{M}|\nabla \chi|_{\omega_{t}}^{2} \operatorname{tr}_{\varphi} \omega_{t} \omega_{\varphi}^{n} \\
& \leq C \int_{M} \operatorname{tr}_{\varphi}\left(\omega_{\varphi}-\sqrt{-1} \partial \bar{\partial} \varphi\right) \omega_{\varphi}^{n} \\
& =C \int_{M}\left(n-\Delta_{\varphi} \varphi\right) \omega_{\varphi}^{n} \leq C,
\end{aligned}
$$


where the first inequality follows from an elementary inequality and the second inequality makes use of the positivity of $\omega_{t}$.

In other words, any smooth cut-off function automatically has uniform $W^{1,2}$ norm with respect to any Kähler metric in any given Kähler class.

Claim 3. We have

$$
\int_{M} f|\nabla \varphi|_{\varphi}^{2} \omega_{t}^{n}=\int_{M}|\nabla \varphi|_{\varphi}^{2} \omega_{\varphi}^{n} \leq C\left(|\varphi|_{L^{\infty}}\right) .
$$

Proof of Claim 3. Let $c$ be the same as in the proof of Claim 1 .

$$
\begin{aligned}
\int_{M}|\nabla \varphi|_{\varphi}^{2} \omega_{\varphi}^{n} & =-\int_{M}(\varphi-c) \Delta_{\varphi} \varphi \omega_{\varphi}^{n} \\
& =\int_{M}(\varphi-c) \operatorname{tr}_{\varphi} \omega_{t} \cdot \omega_{\varphi}^{n}-n \int_{M}(\varphi-c) \omega_{\varphi}^{n} \\
& \leq C .
\end{aligned}
$$

In the last inequality, we have used the fact that $\varphi$ is uniformly bounded.

Claim 4. For any positive $\chi$, the following inequality holds

$$
\int_{0}^{t} \int_{M} \chi f^{2} \operatorname{tr}_{\varphi} \omega_{t} \omega_{t}^{n} d u \leq C_{1} t+C_{2} \sqrt{t}
$$

where both constants depend only on $|\chi|_{L^{\infty}}$ and $|\nabla \chi|_{\omega, L^{\infty}}$.

\section{Proof of Claim 4.}

$$
\begin{aligned}
& \int_{M} \chi f^{2} \operatorname{tr}_{\varphi} \omega_{t} \omega_{t}^{n} \\
= & \int_{M} \chi f \omega_{t} \wedge \omega_{\varphi}^{n-1} \\
= & \int_{M} \chi f \omega_{\varphi}^{n}-\int_{M} \chi f \sqrt{-1} \partial \bar{\partial} \varphi \wedge \omega_{\varphi}^{n-1} \\
\leq & C+\int_{M} \sqrt{-1} \chi \partial f \wedge \bar{\partial} \varphi \wedge \omega_{\varphi}^{n-1}+\int_{M} \sqrt{-1} f \partial \chi \wedge \bar{\partial} \varphi \wedge \omega_{\varphi}^{n-1} \\
\leq & C\left(1+\left(\int_{M}|\nabla f|_{\varphi}^{2} \omega_{\varphi}^{n}\right)^{\frac{1}{2}}\left(\int_{M}|\nabla \varphi|_{\varphi}^{2} \omega_{\varphi}^{n}\right)^{\frac{1}{2}}\right. \\
& \left.+\left(\int_{M}|\nabla \chi|_{\varphi}^{2} \omega_{\varphi}^{n}\right)^{\frac{1}{2}} \cdot\left(\int_{M} f^{2}|\nabla \varphi|_{\varphi}^{2} \omega_{\varphi}^{n}\right)^{\frac{1}{2}}\right) \\
\leq & C\left(1+\left(\int_{M}|\nabla f|_{\varphi}^{2} \omega_{\varphi}^{n}\right)^{\frac{1}{2}}+\left(\int_{M} f^{2}|\nabla \varphi|_{\varphi}^{2} \omega_{\varphi}^{n}\right)^{\frac{1}{2}}\right)
\end{aligned}
$$

We have used Claims 2 and Claim 3 in deriving the last inequality.

Then Claim 4 follows from integrating the above inequality from 0 to $t$ and using Claim 1 and the Schwartz inequality.

A straightforward computation shows

$$
\begin{aligned}
\frac{\partial \log f}{\partial t} & =\operatorname{tr}_{\varphi}\left(\frac{\partial}{\partial t} \omega_{t}\right)+\Delta_{\varphi} \varphi^{\prime}-\operatorname{tr}_{\omega_{t}} \frac{\partial}{\partial t} \omega_{t} \\
& =\Delta_{\varphi} \log f+\operatorname{tr}_{\varphi}\left(-\partial \bar{\partial} h_{\omega_{t}}+\frac{\partial}{\partial t} \omega_{t}\right)-\operatorname{tr}_{\omega_{t}} \frac{\partial}{\partial t} \omega_{t} \\
& \leq \frac{\Delta_{\varphi} f}{f}-\frac{|\nabla f|_{\varphi}^{2}}{f^{2}}+C \operatorname{tr}_{\varphi} \omega_{t}+C .
\end{aligned}
$$


Using this, we deduce

$$
\begin{aligned}
\frac{d}{d t} \int_{M} \chi f^{2} \omega_{t}^{n}= & \int_{M} \chi\left(2 f \frac{\partial f}{\partial t}+f^{2} \operatorname{tr}_{\omega_{t}} \frac{\partial}{\partial t} \omega_{t}\right) \omega_{t}^{n} \\
\leq & 2 \int_{M} \chi f\left(\Delta_{\varphi} f-\frac{|\nabla f|_{\varphi}^{2}}{f}+C f \operatorname{tr}_{\varphi} \omega_{t}+C f\right) \omega_{t}^{n} \\
\leq & -2 \int_{M}(\nabla \chi, \nabla f)_{\varphi} \omega_{\varphi}^{n}+C \int_{M} \chi f^{2} \omega_{t}^{n}+C \int_{M} \chi f^{2} \operatorname{tr}_{\varphi} \omega_{t} \omega_{t}^{n} \\
\leq & 2 \sqrt{\int_{M}|\nabla \chi|_{\varphi}^{2} \omega_{\varphi}^{n}} \sqrt{\int_{M}|\nabla f|_{\varphi}^{2}} \omega_{\varphi}^{n} \\
& +C \int_{M} \chi f^{2} \omega_{t}^{n}+C \int_{M} \chi f^{2} \operatorname{tr}_{\varphi} \omega_{t} \omega_{t}^{n} \\
\leq & C \sqrt{\int_{M}|\nabla f|_{\varphi}^{2} \omega_{\varphi}^{n}}+C \int_{M} \chi f^{2} \omega_{t}^{n}+C \int_{M} \chi f^{2} \operatorname{tr}_{\varphi} \omega_{t} \omega_{t}^{n} .
\end{aligned}
$$

Note that

$$
\int_{M} \chi \cdot f^{2} \omega_{t}^{n} \leq C, \quad \forall t \in[0, T] .
$$

Integrating the above inequality from $t=0$ to $t=t_{i}$ and using Claim 4 , we have

$$
\left.\int_{M} \chi f^{2} \omega_{t}^{n}\right|_{t_{i}} \leq\left.\int_{M} \chi f^{2} \omega_{t}^{n}\right|_{0}+C\left(t_{i}+\sqrt{t_{i}}\right) .
$$

In deriving the above inequality, we used the fact that $\varphi_{0}$ is smooth. For general $\varphi_{0}$ as given, applying the above to $\varphi\left(s, t_{i}\right)$ for any $s>0$ and then taking the limit as $s$ tends to 0 , we get for any $\varphi\left(t_{i}\right)$,

$$
\int_{M} \chi\left(\frac{\omega_{\varphi\left(t_{i}\right)}^{n}}{\omega_{t_{i}}^{n}}\right)^{2} \omega_{t_{i}}^{n} \leq \int_{M} \chi\left(\frac{\omega_{\varphi_{0}}^{n}}{\omega^{n}}\right)^{2} \omega^{n}+C\left(t_{i}+\sqrt{t_{i}}\right) .
$$

Here one can use Fatou's Lemma and the strong convergence for the measure at initial time.

On the other hand, using the assumption that $\varphi\left(t_{i}\right)$ converges to $\tilde{\varphi}_{0}$, we can show that $\frac{\omega_{\varphi\left(t_{i}\right)}^{n}}{\omega_{t_{i}}^{n}}$ converges weakly to $\frac{\omega_{\varphi_{0}}^{n}}{\omega^{n}}$ in $L^{2}(M, \omega)$. Then by taking $t_{i}$ to 0 , we can justify the convergence of integration in a similar way and have

$$
\int_{M} \chi\left(\frac{\omega_{\tilde{\varphi}_{0}}^{n}}{\omega^{n}}\right)^{2} \omega^{n} \leq \int_{M} \chi\left(\frac{\omega_{\varphi_{0}}^{n}}{\omega^{n}}\right)^{2} \omega^{n}
$$

Since this holds for any non-negative smooth cut-off function $\chi$, we have

$$
0 \leq \frac{\omega_{\tilde{\varphi}_{0}}^{n}}{\omega^{n}} \leq \frac{\omega_{\varphi_{0}}^{n}}{\omega^{n}}
$$

a.e. in $M$. However,

$$
\int_{M} \frac{\omega_{\tilde{\varphi}_{0}}^{n}}{\omega^{n}} \omega^{n}=\int_{M} \frac{\omega_{\varphi_{0}}^{n}}{\omega^{n}} \omega^{n}=\operatorname{vol}(M) !
$$

It follows that

$$
\omega_{\tilde{\varphi}_{0}}^{n} \equiv \omega_{\varphi_{0}}^{n}
$$

in $L^{2}(M, \omega)$. The uniqueness of the Monge-Ampère equation for the $C^{0}$ solution by Kolodziej as in [26] implies that $\tilde{\varphi}_{0}=\varphi_{0}$. Since $\left\{t_{i}\right\}$ is any sequence going to 0 , we have proved that $\varphi(t)$ converges to $\varphi_{0}$ as $t$ tends to 0 . 
Furthermore, we have

$$
\int_{M} \chi\left(\frac{\omega_{\tilde{\varphi}_{0}}^{n}}{\omega^{n}}\right)^{2} \omega^{n}=\lim _{i \rightarrow \infty} \int_{M} \chi\left(\frac{\omega_{\varphi_{i}}^{n}}{\omega^{n}}\right)^{2} \omega^{n} .
$$

Together with weak convergence, we conclude that $\frac{\omega_{\varphi_{i}}^{n}}{\omega^{n}}$ converges strongly to $\frac{\omega_{\varphi_{0}}^{n}}{\omega^{n}}$ in $L^{2}(M, \omega)$. Hence Lemma 2.5 is proved.

So far, we have shown that with appropriate assumptions on the volume form, the solution of the weak Kähler-Ricci flow really goes (back) to the initial data as $t \rightarrow 0$.

\section{Higher ORDER ESTIMATES}

In this section, we prove the regularity of the weak Ricci flow under appropriate assumptions on the initial Kähler potential $\varphi_{0}$.

3.1. The $C^{1,1}$ case. In this subsection, we want to prove the following Laplacian estimates.

Proposition 3.1 ([9]). If $\varphi_{0} \in C l_{L^{\infty}} P(M, \omega)$ is $C^{1,1}$-bounded, then

$$
0 \leq \omega_{t}+\sqrt{-1} \partial \bar{\partial} \varphi(t) \leq C, \quad \forall t \in[0, T] .
$$

In other words, $\varphi(t)$ is uniformly $C^{1,1}$-bounded. Moreover, $\frac{\omega_{\varphi^{n}}(t)}{\omega^{n}}$ converges to $\frac{\omega_{\varphi_{0}}^{n}}{\omega^{n}}$ strongly in $L^{2}$ space as $t \rightarrow 0$.

Proof. The $C^{1,1}$ assumption on $\varphi_{0}$ gives an $L^{\infty}$ (upper) bound for the volume form. As with Lemma 2.5 it suffices to prove the Laplacian estimate.

Let $\varphi_{0}(s)$ be the smooth approximations of $\varphi_{0}$ and let $\varphi(s, t)$ be the associated solutions as before. Since $\varphi_{0}$ is in $C^{1,1}$, we can arrange $\varphi_{0}(s)$ with uniformly bounded $C^{1,1}$-norms. Thus, we only need to bound $\varphi(s, t)$ uniformly in $C^{1,1}$. For simplicity, $\varphi$ stands for $\varphi(s, \cdot)$ below. By Lemma 2.2 , it is uniformly bounded.

We first derive a pointwise uniform upper bound on the volume form for $t \in[0, T]$ as follows:

$$
\begin{aligned}
\frac{\partial}{\partial t}\left(\frac{\partial \varphi}{\partial t}\right) & \leq \Delta\left(\frac{\partial \varphi}{\partial t}\right)+C\left(1+\operatorname{tr}_{\varphi} \omega_{t}\right) \\
& \leq \Delta\left(\frac{\partial \varphi}{\partial t}-C \varphi\right)+C+C\left(\operatorname{tr}_{\varphi}(\sqrt{-1} \partial \bar{\partial} \varphi)+\operatorname{tr}_{\varphi} \omega_{t}\right) \\
& \leq \Delta\left(\frac{\partial \varphi}{\partial t}-C \varphi\right)+C+n \\
& \leq \Delta\left(\frac{\partial \varphi}{\partial t}-C \varphi\right)+C+C \varphi .
\end{aligned}
$$

It can be reformulated as

$$
\frac{\partial}{\partial t}\left(\frac{\partial \varphi}{\partial t}-C \varphi\right) \leq \Delta\left(\frac{\partial \varphi}{\partial t}-C \varphi\right)+C-C\left(\frac{\partial \varphi}{\partial t}-C \varphi\right) .
$$

Applying the Maximum Principle, one can easily derive a uniform upper bound for $\frac{\partial \varphi}{\partial t}-C \varphi$. Since $\varphi$ is uniformly bounded, we get a uniform upper bound on $\frac{\partial \varphi}{\partial t}$, so the volume form is uniformly bounded from above.

By standard computation as in 32, we have

$$
e^{C \varphi}\left(\Delta_{\omega_{\varphi}}-\frac{\partial}{\partial t}\right)\left(e^{-C \varphi} \operatorname{tr}_{\omega_{t}}\left(\omega_{\varphi}\right)\right) \geq-C+\left(C \frac{\partial \varphi}{\partial t}-C\right) \operatorname{tr}_{\omega_{t}}\left(\omega_{\varphi}\right)+C e^{-\frac{1}{n-1} \frac{\partial \varphi}{\partial t}} \operatorname{tr}_{\omega_{t}}\left(\omega_{\varphi}\right)^{\frac{n}{n-1}} .
$$


Suppose that the maximum of $e^{-C \varphi} \operatorname{tr}_{\omega_{t}}\left(\omega_{\varphi}\right)$ is attained at some $(p, t)$ in $M \times$ $[0, T]$. At that point, assuming $t>0$ without loss of generality, we have

$$
0 \geq-C+\left(C \frac{\partial \varphi}{\partial t}-C\right) \operatorname{tr}_{\omega_{t}}\left(\omega_{\varphi}\right)+C e^{-\frac{1}{n-1} \frac{\partial \varphi}{\partial t}} \operatorname{tr}_{\omega_{t}}\left(\omega_{\varphi}\right)^{\frac{n}{n-1}} .
$$

Using the upper bound for $\frac{\partial \varphi}{\partial t}$, we have

$$
\begin{aligned}
\operatorname{tr}_{\omega_{t}}\left(\omega_{\varphi}\right)^{\frac{n}{n-1}} & \leq C e^{\frac{1}{n-1} \frac{\partial \varphi}{\partial t}}+C e^{\frac{1}{n-1} \frac{\partial \varphi}{\partial t}} \operatorname{tr}_{\omega_{t}}\left(\omega_{\varphi}\right)-C \frac{\partial \varphi}{\partial t} e^{\frac{1}{n-1} \frac{\partial \varphi}{\partial t}} \operatorname{tr}_{\omega_{t}}\left(\omega_{\varphi}\right) \\
& \leq C+C \operatorname{tr}_{\omega_{t}}\left(\omega_{\varphi}\right) .
\end{aligned}
$$

This implies a uniform upper bound of $\operatorname{tr}_{\omega_{t}}\left(\omega_{\varphi}\right)$ at $(p, t)$. Since $\varphi$ is uniformly bounded, it follows that this trace is uniformly bounded. The proposition is thus proved.

3.2. The $L^{\infty}$ case. We are going to finish proving Theorems 1.1 and 1.3 in this subsection. The estimates will be local for $t>0$ under the weaker assumption that the initial Kähler potential is merely bounded.

Let us point out that in this case, one can still define weak flow using smooth decreasing approximation of the initial bounded Kähler potential 2 These approximation flows have potentials decreasing to the weak flow pointwisely. Moreover, for two choices of such approximations, it is not hard to see that the limits would be the same, because the one-sided relation at the initial time will be preserved along the flow. All these can be justified by applying the Maximum Principle as in Lemma 2.1. Thus we have uniqueness of the weak flow. This also makes sure that what we discuss below is the same weak flow as before when there was more regularity assumptions.

Recall that $\omega_{t}$ is a smooth family of Kähler metrics with $\left[\omega_{t}\right]=[\omega]-t c_{1}(M)$ and $\omega_{\varphi}=\omega_{t}+\sqrt{-1} \partial \bar{\partial} \varphi$. Moreover, the Kähler-Ricci flow is reduced to the following equation for $\varphi$ :

$$
\frac{\partial \varphi}{\partial t}=\log \frac{\left(\omega_{t}+\sqrt{-1} \partial \bar{\partial} \varphi\right)^{n}}{\omega_{t}^{n}}-h_{\omega_{t}}, \varphi(0, \cdot)=\varphi_{0}
$$

$\varphi(t)$ is obtained by taking the limit of $\varphi(s, t)$ as $s$ tends to 0 . In order to prove the theorem, we only need to get uniform estimates for higher order derivatives of $\varphi(s, t)$ for all $s>0$ small. As before, for simplicity, we can simply assume that $\varphi_{0}$ is smooth (as one of the $\varphi(s, 0)$ ).

Choose any interval $\left[t_{1}, t_{0}\right]$ with $T>t_{1}>t_{0}>0$. By Lemmas 2.2 and 2.3, we have the uniform bound for $\varphi$ and $\frac{\partial \varphi}{\partial t}$ for $t \in\left[t_{0}, t_{1}\right]$.

Now we derive the Laplacian and higher order derivative estimates by the standard methods (see [32]; also see [6] or [34]). The estimates may depend on $t_{0}$.

As in the last subsection, we have

$$
e^{C \varphi}\left(\Delta_{\omega_{\varphi}}-\frac{\partial}{\partial t}\right)\left(e^{-C \varphi_{\operatorname{tr}_{\omega_{t}}}}\left(\omega_{\varphi}\right)\right) \geq-C-C \operatorname{tr}_{\omega_{t}}\left(\omega_{\varphi}\right)+C \operatorname{tr}_{\omega_{t}}\left(\omega_{\varphi}\right)^{\frac{n}{n-1}},
$$

where the bound on $\frac{\partial \varphi}{\partial t}$ for $t \in\left[t_{0}, t_{1}\right]$ has been used. The bound for $e^{-C \varphi} \operatorname{tr}_{\omega_{t}}\left(\omega_{\varphi}\right)$ at $t-t_{0}=0$ is not available. So we consider the following quantity instead:

$$
\left(t-t_{0}\right)^{n-1} e^{-C \varphi} \operatorname{tr}_{\omega_{t}}\left(\omega_{\varphi}\right) .
$$

\footnotetext{
${ }^{2}$ This is provided by the result in [3] as a simple version of the more general result in [16] for this case.
} 
Straightforward computation then gives

$$
\begin{aligned}
& e^{C \varphi}\left(\Delta_{\omega_{\varphi}}-\frac{\partial}{\partial t}\right)\left(\left(t-t_{0}\right)^{n-1} e^{\left.-C \varphi_{\operatorname{tr}_{\omega_{t}}}\left(\omega_{\varphi}\right)\right)}\right. \\
& \quad \geq\left(t-t_{0}\right)^{n-1}\left(-C-C \operatorname{tr}_{\omega_{t}}\left(\omega_{\varphi}\right)+C \operatorname{tr}_{\omega_{t}}\left(\omega_{\varphi}\right)^{\frac{n}{n-1}}\right)-(n-1)\left(t-t_{0}\right)^{n-2} \operatorname{tr}_{\omega_{t}}\left(\omega_{\varphi}\right) .
\end{aligned}
$$

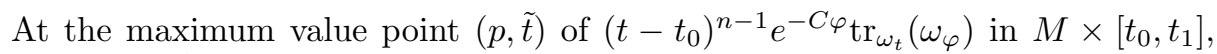
where clearly $\tilde{t}>t_{0}$,

$$
\begin{aligned}
0 & \geq\left(\tilde{t}-t_{0}\right)^{n-1}\left(-C-C \operatorname{tr}_{\omega_{t}}\left(\omega_{\varphi}\right)+C \operatorname{tr}_{\omega_{t}}\left(\omega_{\varphi}\right)^{\frac{n}{n-1}}\right)-(n-1)\left(\tilde{t}-t_{0}\right)^{n-2} \operatorname{tr}_{\omega_{t}}\left(\omega_{\varphi}\right) \\
& =-C\left(\tilde{t}-t_{0}\right)^{n-1}-(C \tilde{t}+C)\left(\tilde{t}-t_{0}\right)^{n-2} \operatorname{tr}_{\omega_{t}}\left(\omega_{\varphi}\right)+C\left(\tilde{t}-t_{0}\right)^{n-1} \operatorname{tr}_{\omega_{t}}\left(\omega_{\varphi}\right)^{\frac{n}{n-1}}
\end{aligned}
$$

Multiply both sides by $\tilde{t}-t_{0}$ and reformulate the above to be

$$
0 \geq-C\left(\tilde{t}-t_{0}\right)^{n}-(C \tilde{t}+C)\left(\tilde{t}-t_{0}\right)^{n-1} \operatorname{tr}_{\omega_{t}}\left(\omega_{\varphi}\right)+C\left(\left(\tilde{t}-t_{0}\right)^{n-1} \operatorname{tr}_{\omega_{t}}\left(\omega_{\varphi}\right)\right)^{\frac{n}{n-1}} .
$$

So we get

$$
\left(\tilde{t}-t_{0}\right)^{n-1} \operatorname{tr}_{\omega_{t}}\left(\omega_{\varphi}\right) \leq C .
$$

Thus at $(p, \tilde{t})$, using the bound for $\varphi$,

$$
\left(\tilde{t}-t_{0}\right)^{n-1} e^{-C \varphi} \operatorname{tr}_{\omega_{t}}\left(\omega_{\varphi}\right) \leq C .
$$

It follows that for all $t \in\left[t_{0}, t_{1}\right]$,

$$
\left(t-t_{0}\right)^{n-1} e^{-C \varphi} \operatorname{tr}_{\omega_{t}}\left(\omega_{\varphi}\right) \leq C, \text { on } M,
$$

which implies that

$$
\operatorname{tr}_{\omega_{t}}\left(\omega_{\varphi}\right) \leq \frac{C}{\left(t-t_{0}\right)^{n-1}} .
$$

This together with the volume lower bound gives the uniform metric bound locally away from $t=0$.

Next, we can get the third order estimate in a similar fashion. We have a similar inequality as in [6]. Of course, in order to do this, we still translate the time to guarantee the uniform metric bound. Let us say that the time is translated so that the original time $t=t_{0}>0$ is now the new initial time $t=0$. The inequality is as follows, for $S=g_{\varphi}^{i \bar{j}} g_{\varphi}^{k \bar{l}} g_{\varphi}^{\lambda \bar{\eta}} \varphi_{i \bar{l} \lambda} \varphi_{\bar{j} k \bar{\eta}}$ :

$$
\left(\Delta_{\varphi}-\frac{\partial}{\partial t}\right) S \geq-C \cdot S-C .
$$

As in [32], we also have

$$
\left(\Delta_{\varphi}-\frac{\partial}{\partial t}\right) \Delta_{\omega_{t}} \varphi \geq C \cdot S-C .
$$

From the above differential inequality for $S$, as in the Laplacian estimate, we consider $t S$ instead:

$$
\left(\Delta_{\varphi}-\frac{\partial}{\partial t}\right)(t S) \geq-C t \cdot S-C t-S
$$

Choosing $A>0$ large enough, one has, for $t \in[0, T]$,

$$
\left(\Delta_{\varphi}-\frac{\partial}{\partial t}\right)\left(t S+A \Delta_{\omega_{t}} \varphi\right) \geq C \cdot S-C .
$$

The function $t S+A \Delta_{\omega_{t}} \varphi$ is uniformly bounded at the (new) initial time, $t=0$. By the Maximum Principle, if its maximum value is achieved at some point in $M$ 
and $t>0$, then $S$ is bounded there. Consequently, the whole function $t S+A \Delta_{\omega_{t}} \varphi$ is also bounded there. So we finally conclude that

$$
t S+A \Delta_{\omega_{t}} \varphi \leq C,
$$

which implies

$$
S \leq \frac{C}{t}
$$

This provides a local $C^{2, \alpha}$ bound for $\varphi$. After this, higher order estimates follow from the standard argument.

Therefore, by the Ascoli-Arzela Theorem, we have proved that the weak flow considered so far is actually smooth in $(0, T]$. Theorem 1.1 follows from this and the second order estimate in Subsection 3.1. Theorem 1.3 follows from this and Lemma 2.5.

\section{4. $C^{1,1} \mathrm{~K}$-ENERGY MINIMIZER}

In an earlier paper [9] where the present work was initiated, the first two named authors proved that

Proposition 4.1. In any Kähler class, the volume form of any $C^{1,1} \mathrm{~K}$-energy minimizer belongs to $H^{1,2}(M, \omega)$.

By Lemma 2.5 and Proposition 3.1. we can prove a stronger result.

Theorem 4.2. The $C^{1,1}$ minimizer of the K-energy functional in any canonical Kähler class with positive first Chern class is necessarily smooth.

Proof. Let $\phi_{0}$ be a $C^{1,1}$ Kähler potential which minimizes the K-energy functional in the canonical Kähler class. According to the results proved in the preceding sections, there is a unique smooth Kähler-Ricci flow $\varphi(t)(t>0)$ such that

$$
\frac{\partial \varphi}{\partial t}=\log \frac{\omega_{\varphi}^{n}}{\omega^{n}}+\varphi-h_{\omega} .
$$

Moreover, $\lim _{t \rightarrow 0} \frac{\omega_{\varphi}^{n}}{\omega^{n}}=\frac{\omega_{\varphi_{0}}^{n}}{\omega^{n}}$ strongly in $L^{2}\left(M, g_{0}\right)$ and the Kähler potential $\varphi(t)$ converges to $\varphi_{0}$ strongly in $C^{1, \alpha}(M)$ for any $\alpha \in(0,1)$. Since the K-energy is decreasing along the Kähler-Ricci flow, we have

$$
\limsup _{t \rightarrow 0^{+}} \mathbf{E}(\varphi(t)) \leq \mathbf{E}\left(\varphi_{0}\right)=\inf _{\phi \in \mathcal{H}} \mathbf{E}(\phi) .
$$

Since the K-energy is non-increasing for $\varphi(t)(t>0)$, then for any $t>0$, we have

$$
\inf _{\phi \in \mathcal{H}} \mathbf{E}(\phi) \leq \mathbf{E}(\varphi(t)) \leq \inf _{\phi \in \mathcal{H}} \mathbf{E}(\phi) .
$$

In other words,

$$
\mathbf{E}(\varphi(t))=\inf _{\phi \in \mathcal{H}} \mathbf{E}(\phi), \quad t>0 .
$$

Since $\omega_{\varphi(t)}$ is a smooth Kähler metric, this means that the scalar curvature of $\omega_{\varphi(t)}(t>0)$ must be constant. Consequently, $\omega_{\varphi(t)}$ is a Kähler-Einstein metric for all $t>0$. This. in turn, implies that $\frac{\partial \varphi}{\partial t}$ is a function of $t$ only. Note that $\varphi_{0}$ is the strong $C^{1, \alpha}$ limit of $\varphi(t)$ as $t \rightarrow 0$. Therefore, $\varphi_{0}-\varphi(t)$ is a constant which depends only on $t$. In other words, $\omega_{\varphi_{0}}$ is also a Kähler-Einstein metric and the theorem is then proved. 


\section{REFERENCES}

1. S. Bando and T. Mabuchi. Uniqueness of Einstein Kähler metrics modulo connected group actions. Algebraic geometry, Sendai, 1985, 11-40, Adv. Stud. Pure Math., 10, North-Holland, Amsterdam, 1987. MR946233 (89c:53029)

2. E. Bedford and B. A. Taylor. A new capacity for plurisubharmonic functions. Acta Math. 149 (1982), no. 1-2, 1-40. MR674165 (84d:32024)

3. Z. Blocki and S. Kolodziej. On regularization of plurisubharmonic functions on manifolds. Proc. Amer. Math. Soc. 135 (2007), no. 7, 2089-2093 (electronic). MR2299485|(2008a:32029)

4. E. Calabi. Extremal Kähler metrics. In Seminar on Differential Geometry, volume 16 of 102, pages 259-290. Ann. of Math. Studies, University Press, 1982. MR645743 (83i:53088)

5. E. Calabi. Extremal Kähler metrics, II. In Differential geometry and Complex analysis, pages 96-114. Springer, 1985. MR780039 (86h:53067)

6. H. D. Cao. Deformation of Kähler metrics to Kähler-Einstein metrics on compact Kähler manifolds. Invent. Math. 81 (1985), no. 2, 359-372. MR799272 (87d:58051)

7. X. X. Chen. Weak limits of Riemannian metrics in surfaces with integral curvature bound. Calc. Var. Partial Differential Equations 6 (1998), no. 3, 189-226. MR1614627 (99k:53087)

8. X. X. Chen. Space of Kähler metrics. Journal of Differential Geometry, 56(2):189-234, 2000. MR.1863016 (2003b:32031)

9. X. X. Chen and G. Tian. Foliation by holomorphic discs and its application in Kähler geometry, math.DG/0507148. Submitted.

10. X. X. Chen. Space of Kähler metrics (III) - On the greatest lower bound of the Calabi energy and the lower bound of geodesic distance. Invent. Math. 175 (2009), no. 3, 453-503. MR2471594 (2010b:32033)

11. X. X. Chen and W. Y. Ding. Ricci flow on surfaces with $L^{\infty}$ initial metrics. Ricci flow on surfaces with degenerate initial metrics. J. Partial Differential Equations 20 (2007), no. 3, 193-202. MR2348984 (2008g:53081)

12. X. X. Chen and G. Tian. Ricci flow on Kähler-Einstein surfaces. Invent. Math. 147 (2002), no. 3, 487-544. MR.1893004 (2003c:53095)

13. X. X. Chen and G. Tian. Ricci flow on Kähler-Einstein manifolds. Duke. Math. J. 131 (2006), no. 1, 17-73. MR2219236 (2007c:53083)

14. B. Chow. The Ricci flow on the 2-sphere. J. Differential Geom. 33 (1991), no. 2, 325-334. MR.1094458 (92d:53036)

15. B. Chow, P. Lu and L. Ni. Hamilton's Ricci flow. Graduate Studies in Mathematics, 77. American Mathematical Society, Providence, RI; Science Press, New York, 2006. xxxvi+608 pp. ISBN: 978-0-8218-4231-7; 0-8218-4231-5 MR2274812 (2008a:53068)

16. J. -P. Demailly and M. Paun. Numerical characterization of the Kähler cone of a compact Kähler manifold. Ann. of Math. 159 (2004), 1247-1274. MR2113021(2005i:32020)

17. W.Y. Ding and G. Tian. Kähler-Einstein metrics and the generalized Futaki invariant. Invent. Math. 110 (1992), no. 2, 315-335. MR.1185586 (93m:53039)

18. S. K. Donaldson. Scalar curvature and projective embeddings, II. Q. J. Math. 56 (2005), no. 3, 345-356. MR2161248 (2006f:32033)

19. S. K. Donaldson. Lower bounds on the Calabi functional. math.DG/0506501.

20. R. Hamilton. Three-manifolds with positive Ricci curvature. J. Diff. Geom. 17:255-306, 1982. MR664497 (84a:53050)

21. R. Hamilton. The formation of singularities in the Ricci flow, volume II. Internat. Press, 1993.

22. G. Huisken. Ricci deformation of the metric on a Riemannian manifold. J. Differential Geom. 21 (1985), no. 1, 47-62. MR806701 (86k:53059)

23. T. Mabuchi. K-energy maps integrating Futaki invariants. Tohoku Math. J. 38 (1986), 575593. MR867064 (88b:53060)

24. T. Mabuchi. An energy-theoretic approach to the Hitchin-Kobayashi correspondence for manifolds. I. Invent. Math. 159 (2005), no. 2, 225-243. MR2116275 (2005m:32047)

25. G. Perelman. The entropy formula for the Ricci flow and its geometric applications, http://arxiv.org/abs/math.DG/0211159.

26. S. Kolodziej. The complex Monge-Ampere equation and pluripotential theory. Mem. Amer. Math. Soc. 178 (2005), no. 840, x+64 pp. MR2172891 (2006d:32054) 
27. S. Kolodziej. Hölder continuity of solutions to the complex Monge-Ampère equation with the right hand side in $L^{p}$. The case of compact Kähler manifolds. Math. Ann. 342 (2008), no. 2, 379-386. MR2425147 (2009g:32079)

28. J. Song and G. Tian. The Kähler-Ricci flow on surfaces of positive Kodaira dimension. Inventiones Math. 170 (2007), no. 3, 609-653. MR2357504 (2008m:32044)

29. G. Tian. Kähler-Einstein metrics with positive scalar curvature. Invent. Math. 130 (1997), no. 1, 1-37. MR 1471884 (99e:53065)

30. G. Tian and X. H. Zhu. Convergence of Kähler-Ricci flow. J. Amer. Math. Soc. 20 (2007), no. 3, 675-699. MR2291916 (2007k:53107)

31. G. Tian and Z. Zhang. On the Kähler-Ricci flow on projective manifolds of general type. Chinese Annals of Mathematics - Series B, Volume 27, Number 2, 179-192. MR2243679 (2007c:32029)

32. S. T. Yau. On the Ricci curvature of a compact Kähler manifold and the complex MongeAmpère equation, I. Comm. Pure Appl. Math. 31 (1978), no. 3, 339-411. MR480350 (81d:53045)

33. Z. Zhang. On Degenerate Monge-Ampère Equations over Closed Kähler Manifolds. Int. Math. Res. Not. 2006, Art. ID 63640, 18 pp. MR2233716 (2007b:32058)

34. Z. Zhang. Degenerate Monge-Ampere Equations over Projective Manifolds. Ph.D. Thesis at MIT, 2006.

Department of Mathematics, University of Wisconsin, Madison, Wisconsin 53706

E-mail address: xiu@math.wisc.edu

Department of Mathematics, Princeton University, Princeton, New Jersey 08544

E-mail address: tian@math.princeton.edu

Department of Mathematics, University of Michigan, Ann Arbor, Michigan 48109

E-mail address: zhangou@umich.edu

Current address: School of Mathematics and Statistics, University of Sydney, Carslaw Building, Sydney, NSW 2006, Australia

E-mail address: zhangou@maths.usyd.edu.au 